\title{
Article
}

\section{Optimizing the Pharmacological and Optical Dosimetry for Photodynamic Therapy with Methylene Blue and Nanoliposomal Benzoporphyrin on Pancreatic Cancer Spheroids}

\author{
Tristan Le Clainche, Nazareth Milagros Carigga Gutierrez (D), Núria Pujol-Solé, Jean-Luc Coll (D) \\ and Mans Broekgaarden * (D)
}

check for

updates

Citation: Le Clainche, T.; Carigga Gutierrez, N.M.; Pujol-Solé, N.; Coll, J.-L.; Broekgaarden, M. Optimizing the Pharmacological and Optical Dosimetry for Photodynamic Therapy with Methylene Blue and Nanoliposomal Benzoporphyrin on Pancreatic Cancer Spheroids. Onco 2022, 2, 19-33. https://doi.org/ $10.3390 /$ onco2010002

Academic Editor: Fred Saad

Received: 15 December 2021

Accepted: 2 January 2022

Published: 7 January 2022

Publisher's Note: MDPI stays neutral with regard to jurisdictional claims in published maps and institutional affiliations.

Copyright: (C) 2022 by the authors. Licensee MDPI, Basel, Switzerland. This article is an open access article distributed under the terms and conditions of the Creative Commons Attribution (CC BY) license (https:// creativecommons.org/licenses/by/ $4.0 /)$.
Institute for Advanced Biosciences, Grenoble-Alpes University, INSERM U1209, CNRS UMR 5309, 38700 Grenoble, France; Tristan.le-clainche@univ-grenoble-alpes.fr (T.L.C.); nazareth.carigga-gutierrez@univ-grenoble-alpes.fr (N.M.C.G.); nuria.pujol-sole@etu.univ-grenoble-alpes.fr (N.P.-S.); jean-luc.coll@univ-grenoble-alpes.fr (J.-L.C.)

* Correspondence: mans.broekgaarden@univ-grenoble-alpes.fr

Simple Summary: Photodynamic therapy is a promising cancer treatment, which relies on a multitude of pharmacological and optical parameters. In this study, we investigated the PDT efficacy of methylene blue and nanoliposomal benzoporphyrin on PANC-1 and MIA PaCa-2 spheroids and the impact of the photosensitizer dose, the light dose, and the fluence rate. We demonstrate that maximizing the PDT effects requires the optimization of both the pharmacological and optical dosimetry in a photosensitizer and cancer model-dependent manner.

\begin{abstract}
Photodynamic therapy (PDT) is a cancer treatment that relies on the remote-controlled activation of photocatalytic dyes (photosensitizers) in cancer tissues. To effectively treat cancer, a variety of pharmacological and optical parameters require optimization, which are dependent on the photosensitizer type. As most photosensitizers are hydrophobic molecules, nanoliposomes are frequently used to increase the biocompatibility of these therapeutics. However, as nanoliposomes can influence the therapeutic performance of photosensitizers, the most suitable treatment parameters need to be elucidated. Here, we evaluate the efficacy of PDT on spheroid cultures of PANC-1 and MIA PaCa-2 pancreatic cancer cells. Two strategies to photosensitize the pancreatic microtumors were selected, based on either nanoliposomal benzoporphyrin derivative (BPD), or non-liposomal methylene blue (MB). Using a comprehensive image-based assay, our findings show that the PDT efficacy manifests in distinct manners for each photosensitizer. Moreover, the efficacy of each photosensitizer is differentially influenced by the photosensitizer dose, the light dose (radiant exposure or fluence in $\mathrm{J} / \mathrm{cm}^{2}$ ), and the dose rate (fluence rate in $\mathrm{mW} / \mathrm{cm}^{2}$ ). Taken together, our findings illustrate that the most suitable light dosimetry for PDT strongly depends on the selected photosensitization strategy. The PDT dose parameters should therefore always be carefully optimized for different models of cancer.
\end{abstract}

Keywords: photodynamic therapy; photosensitizer; pancreatic cancer; nanoliposome; optical dosimetry

\section{Introduction}

In recent years, research on photodynamic therapy (PDT) has validated its utility in oncology, as it has been demonstrated to be not only important in the treatment of non-melanoma skin cancer [1] but also as a treatment option for head-and-neck cancer [2,3], lung cancer [4,5] and prostate cancer [6,7]. In addition, it has shown clinical potential for application in gastrointestinal cancer [8,9], pancreatic cancer [10-12] and breast cancer [13]. As a cancer treatment modality, PDT involves the administration of small molecules called photosensitizers, which usually are pharmaceuticals that absorb visible or near-infrared light. 
These photosensitizers induce cytotoxicity within the tumor tissue through photochemical production of cytotoxic reactive oxygen species (ROS) [11,14-16].

Compared to radiotherapy and chemotherapy, PDT is rather complex, and requires the optimization of various parameters. Among the parameters that influence PDT outcomes we can list: (1) the type of photosensitizer, (2) the photosensitizer concentration, (3) the light energy delivered to the target tissue or light dose (given here as radiant exposure in $\mathrm{J} / \mathrm{cm}^{2}$ ), (4) the fluence rate at which the light is delivered (given in $\mathrm{mW} / \mathrm{cm}^{2}$ ), and (5) the sensitivity of tissue to PDT [17-22]. Over the years, the influence of these parameters on PDT outcomes has been investigated in various contexts. For example, for the prephotosensitizer 5-aminolevulinic acid, it was shown that PDT with a radiant exposure of $100 \mathrm{~J} / \mathrm{cm}^{2}$ given at either a low or high light dose rate of $100 \mathrm{~mW} / \mathrm{cm}^{2}$ or $25 \mathrm{~mW} / \mathrm{cm}^{2}$ to melanoma cells implanted in the dorsal skin of hamsters caused a complete remission of tumors only when exposed to PDT at $100 \mathrm{~mW} / \mathrm{cm}^{2}$ [23]. In a study where TB4C spheroids were subjected to 5-aminolevolunic acid-PDT demonstrated that fluence rates below $25 \mathrm{~mW} / \mathrm{cm}^{2}$ were shown to be more effective, compared to PDT with higher fluence rates. An exposure of $50 \mathrm{~J} / \mathrm{cm}^{2}$ reduced spheroid survival to $66 \%$ when using $25 \mathrm{~mW} / \mathrm{cm}^{2}$ as fluence rate, while fluence rates of 0.8 and $5 \mathrm{~mW} / \mathrm{m}^{2}$ reduced spheroid survival to $40 \%$. Moreover, when TB4C intracranial glioma tumors in rats were subjected to PDT with low fluence rates, the treatment did not repress tumor growth but demonstrated that $\mathrm{O}_{2}$ supply was enough to yield necrosis in the tumor core [24].

The most suitable PDT parameters for a given model are thus highly dependent on the type of photosensitizer. As many photosensitizers are highly hydrophobic porphyrins or phthalocyanines, nanoliposomes are frequently used as nanocarriers to render such photosensitizers more biocompatible [25-27]. As nanoparticular encapsulation alters key pharmaceutical parameters such as uptake kinetics, intracellular, and intratumor localization, it may complicate the refinement of the PDT dosimetry. For example, whether the PDT dose parameters optimized for free BPD [28] are similar to liposomal BPD (e.g., Visudyne) remains unclear. Moreover, many photosensitizers, including BPD, have multiple absorption peaks at which they can be excited, and the excitation source may also impact the PDT efficacy. For other common photosensitizers, the most suitable PDT dose parameters remain still largely uninvestigated. For example, methylene blue (MB) is a non-porphyrin photosensitizer widely used for anti-microbial PDT in dental clinical applications [29-31]. However, recent studies have leveraged its ROS production capacity and its experimental potential in some cancers such as breast cancer [32,33] and colorectal cancer [34], yet how the efficacy is influenced by specific cancer models, MB dose, and optical dosimetry remains sparsely investigated.

In the present study, we evaluate the PDT efficacy of liposomal BPD (L-BPD) and MB using state-of-the-art 96-well LED arrays, with the aim to find the optimal PDT dose parameters and establish the appropriate manner to determine the treatment outcomes. This is studied using spheroid models of pancreatic cancer, the third leading cause of cancer deaths in the U.S. and the fourth most common cause in the EU and the UK [35]. We demonstrate that modulating total radiant exposure and fluence rate strongly impact the PDT outcomes in a photosensitizer-dependent manner. Moreover, the treatment outcomes manifest in distinct manners, depending on the cellular origin of the spheroids and the photosensitizer type. Thus, the rational design of PDT strategies is of highly importance to obtain the most optimal treatment outcomes.

\section{Materials and Methods}

\subsection{Chemicals and Reagents}

Methylene blue (MB, 97\% purity), benzoporphyrin derivative (BPD, 94\% purity), aminophenyl fluorescein (APF), and cholesterol were obtained from Sigma-Aldrich (Sigma Aldrich Chimie, Saint-Quentin-Fallavier, France). The lipids 1,2-distearoyl-sn-glycero3-phosphocholine (DSPC), 1,2-dioleoyl-sn-glycero-3- phosphoethanolamine (DOPE), 1,2distearoyl-sn-glycero-3-phosphoethanolamine-N-[methoxy(polyethylene glycol)-2000] 
(DSPE-PEG) were obtained from Avanti Polar Lipids (Sigma Aldrich). The ROS sensor singlet oxygen sensor green (SOSG) was obtained from Thermo Fisher (Waltham, MA, USA).

\subsection{Liposome Preparation and Characterization}

The lipids DOPE, DSPC, DSPE-PEG and Cholesterol (5:41:4:50 molar ratio in $\mathrm{CHCl}_{3}$ ) were mixed and BPD was added to the mixture at a BPD:lipid molar ratio of $0.008 . \mathrm{CHCl}_{3}$ was evaporated and the lipid cake was hydrated in phosphate-buffered saline (PBS, Gibco) to reach a $5 \mathrm{mM}$ final lipid concentration). Liposomes were formed by sonication during 20 min using the EpiShear cooled sonication platform (Active Motif, Carlsbad, CA, USA) and extruded at $0.2 \mu \mathrm{m}$ using Whatman Anotop membrane filters (GE Healthcare, Chicago, IL, USA). For a better preservation, liposomes were purged with $\mathrm{N}_{2}$ gas (Messer CANgas, Sigma-Aldrich) and stored at $4{ }^{\circ} \mathrm{C}$.

The zeta potential, size and polydispersity index of the L-BPD were determined by electrophoretic light scattering spectroscopy and dynamic light scattering using the ZetaSizer (Malvern Panalytical, Malvern, WS, USA). The L-BPD size and zeta potential were $96.8 \pm 2.05 \mathrm{~nm}$ and $2.6 \pm 0.2 \mathrm{mV}$ respectively. Polydispersity index was $0.130 \pm 0.02$, proving the size homogeneity of liposomes population following extrusion.

\subsection{Detection of $\mathrm{HO} \bullet$ and ${ }^{1} \mathrm{O}_{2}$ Generation}

Reaction mixtures of $10 \mu \mathrm{M}$ photosensitizer (L-BPD or MB) in PBS were prepared in clear-bottom 96-wells plates (Corning, Boulogne-Billancourt, France). The reaction mixtures contained either $10 \mu \mathrm{M}$ APF or SOSG for the detection of $\mathrm{OH}^{\bullet}$ and ${ }^{1} \mathrm{O}_{2}$, respectively. The final volume in the wells was $100 \mu \mathrm{L}$. The reaction mixtures were irradiated using Lumidox Gen II, 96-well LED-arrays (Analytical Sales and Services, Flanders, NJ, USA), emitting at either $420 \mathrm{~nm}\left(78.13 \mathrm{~mW} / \mathrm{cm}^{2}\right)$ or $660 \mathrm{~nm}\left(93.75 \mathrm{~mW} / \mathrm{cm}^{2}\right)$. Fractional illumination was performed, during which the fluorescence emission of APF and SOSG was determined at intermediate radiant exposures until a total dose of $37.5 \mathrm{~J} / \mathrm{cm}^{2}(420 \mathrm{~nm})$ or $45 \mathrm{~J} / \mathrm{cm}^{2}(660 \mathrm{~nm})$ was reached. APF and SOSG fluorescence emission was detected at $\lambda e x=485 \mathrm{~nm}$ and $\lambda \mathrm{em}=520 \mathrm{~nm}$ (FluoStar Omega plate reader, BMG Labtech, Champigny-sur-Marne, France).

\subsection{Cell and Spheroid Culture}

PANC-1 and MIAPaCa-2 pancreatic cancer cell lines were purchased from ATCC and maintained in DMEM culture medium (Sigma-Aldrich) supplemented with $10 \%$ Fetal Bovine Serum (ThermoFisher, Waltham, MA, USA) and a mix of Penicillin and Streptomycin at 100 units $/ \mathrm{mL}$ and $100 \mu \mathrm{g} / \mathrm{mL}$ final concentration. Harvesting was performed using 0.05\% Trypsin-EDTA mix in Phosphate Buffer Saline (ThermoFisher). Both cell lines were characterized mycoplasma-free using Mycoalert detection kit (Lonza, Bâle, Switzerland). Spheroids cultures were performed using black 96-wells Corning Spheroid Microplates (Corning, NY, USA). Five thousand cells per well were seeded in $100 \mu \mathrm{L}$ DMEM medium final volume and culture for $72 \mathrm{~h}$ at $37{ }^{\circ} \mathrm{C}$ and $5 \% \mathrm{CO}_{2}$ before treatment.

\subsection{Determination of Photosensitizer Uptake and Dark Toxicity}

For uptake experiments, BPD containing liposomes were diluted to reach a final concentration of $500 \mu \mathrm{M}$ in fully supplemented culture medium. As BPD was included in a BPD:lipid ratio of 0.008 , the final BDP concentration contained in the liposomes was $4 \mu \mathrm{M}$. MB was prepared at a final concentration of $100 \mu \mathrm{M}$ in fully supplemented culture medium. L-BPD and MB were incubated with 3 days-old PANC- 1 and MIAPaCa- 2 spheroid cultures during 2, 4 and $24 \mathrm{~h}$. Then, the liposome uptake was assessed through the quantification of $\mathrm{MB}$ and BPD emission.

The uptake was quantified by confocal fluorescence microscopy (Zeiss Axio Observer Z1) equipped with a $5 \times$ objective. BPD emission was detected using $\lambda e x=405 \mathrm{~nm}$, $\lambda \mathrm{em}=600-740 \mathrm{~nm}$. MB emission was detected using $\lambda \mathrm{ex}=633 \mathrm{~nm}, \lambda \mathrm{em}=670-750 \mathrm{~nm}$. For the photosensitizers uptake, the acquisition parameters were set by maximizing the 
detector gain on the spheroids that were treated for $24 \mathrm{~h}$. Finally, image analysis and quantification of BPD uptake was performed using a custom-designed MATLAB code previously described by Bulin et al. [36].

\subsection{Determination of PDT Efficacy}

3-days old PANC-1 and MIAPaCa-2 spheroids were subjected to the following treatments: (1) A no treatment control group exposed to blue light $(420 \mathrm{~nm})$, (2) PDT with L-BPD in a concentration range of $0.2-20 \mu \mathrm{M}$ and at a blue light irradiance range of $5-50 \mathrm{~J} / \mathrm{cm}^{2}$ (3) A no treatment control group exposed to red light $(660 \mathrm{~nm})$, (4) PDT with MB in a concentration range of $0.2-20 \mu \mathrm{M}$ and at a red light irradiance range of $5-50 \mathrm{~J} / \mathrm{cm}^{2}$, and (5) a total killing control.

To evaluate the effect of fluence rate, 3-days old PANC-1 and MIAPaCa-2 spheroids were subjected to the following treatments: (1) A no treatment control group exposed to blue light $(420 \mathrm{~nm})$, (2) PDT with $0.2 \mu \mathrm{M} \mathrm{L}-\mathrm{BPD}$ at an irradiance of $50 \mathrm{~J} / \mathrm{cm}^{2}$ and at energies of 78,170 or $235 \mathrm{~mW} / \mathrm{cm}^{2}$, (3) A no treatment control group exposed to red light $(660 \mathrm{~nm})$, (4) PDT with $20 \mu \mathrm{M} \mathrm{MB}$ at a red light irradiance of $50 \mathrm{~J} / \mathrm{cm}^{2}$ and at energies of 95,190 or $280 \mathrm{~mW} / \mathrm{cm}^{2}$, and (5) a total killing control.

Liposome toxicity and the effects of PDT were assessed using in situ live/dead staining with $2 \mu \mathrm{M}$ Calcein AM (ThermoFisher), and $3 \mu \mathrm{M}$ propidium iodide (PI, Sigma Aldrich) prepared in complete medium. Spheroid culture medium was removed before adding $100 \mu \mathrm{L}$ of staining solution to each well. Then, plates were incubated for $60 \mathrm{~min}$ at culture conditions, and subsequent confocal laser scanning fluorescence intensities were detected at $\Lambda \mathrm{ex}=488 \mathrm{~nm} / \kappa \mathrm{em}=520 \mathrm{~nm}$ and $\kappa \mathrm{ex}=520 \mathrm{~nm} / \Lambda \mathrm{em}=559 \mathrm{~nm}$ respectively. No treatment and total killing controls were used to set the acquisition parameters by maximizing the detector gain of $488 \mathrm{~nm}$ and $520 \mathrm{~nm}$ lasers respectively. Subsequent image acquisition and image analysis was performed using MATLAB the CALYPSO image analysis code Necrosis was determined by calculating the percentage of the mean propidium iodide emission intensity in each treatment condition in comparison to th the total killing control (set at $100 \%$ ).

\subsection{Statistical Analysis}

All data was statistically analyzed in Graphpad Prism 7 (San Diego, CA, USA). Data sets were tested for Gaussian distributions using D'Agostino and Pearson omnibus normality tests. Normally distributed data sets were analyzed using a one-way ANOVA and Bonferroni post hoc test for multiple comparisons, whereas non-Gaussian data sets were analyzed using a Kruskal-Wallis and Dunn's post-hoc test for multiple comparison. Statistical significance is indicated with single asterisks $(p \leq 0.05)$, double asterisks $(p \leq 0.01)$ or triple asterisks $(p \leq 0.005)$. All data represents the mean \pm SD. Outlier removal (ROUT method, $Q=10 \%$ ) was performed for all the treatments. Dose responses curves were fitted using non-linear regression (Inhibitor versus normalized response and antagonist versus normalized response). Uptake analyses were performed using one-phase association fits.

\section{Results}

\subsection{The Photodynamic Activity of L-BPD and $M B$}

Prior to the in vitro studies, we first set out to evaluate the photodynamic activity of L-BPD and MB, of which the absorption spectra are given in Figure 1A,D). Upon excitation with $420 \mathrm{~nm}$, minor oxidation of APF and SOSG can be observed in the control and liposome-only conditions (Figure 1B,C). Increased SOSG fluorescence emission is likely the result of ${ }^{1} \mathrm{O}_{2}$ production by blue light. This may similarly have caused increased APF fluorescence emission; although APF is primarily a sensor for $\mathrm{OH}^{\bullet}$, it is known to have minor reactivity to ${ }^{1} \mathrm{O}_{2}$ as well [37]. MB demonstrated no photodynamic activity upon excitation with $420 \mathrm{~nm}$ light, which is in line with the absence of absorption at this wavelength. In contrast, L-BPD induced significant APF oxidation, indicating substantial production of $\mathrm{OH}^{\bullet}$. A significant increase in SOSG oxidation was also detected, albeit 
to a lesser extent. Upon $660 \mathrm{~nm}$ irradiation, no APF or SOSG fluorescence emission was detected in solutions containing PBS or liposomes only (Figure 1E,F). MB showed a clear, light dose-dependent increase in APF and SOSG fluorescence emission, indicating that in PBS, MB generates both $\mathrm{OH}^{\bullet}$ and ${ }^{1} \mathrm{O}_{2}$. Taken together, the results clearly demonstrate that both L-BPD and MB are potent photosensitizers when excited with the appropriate wavelength of light.
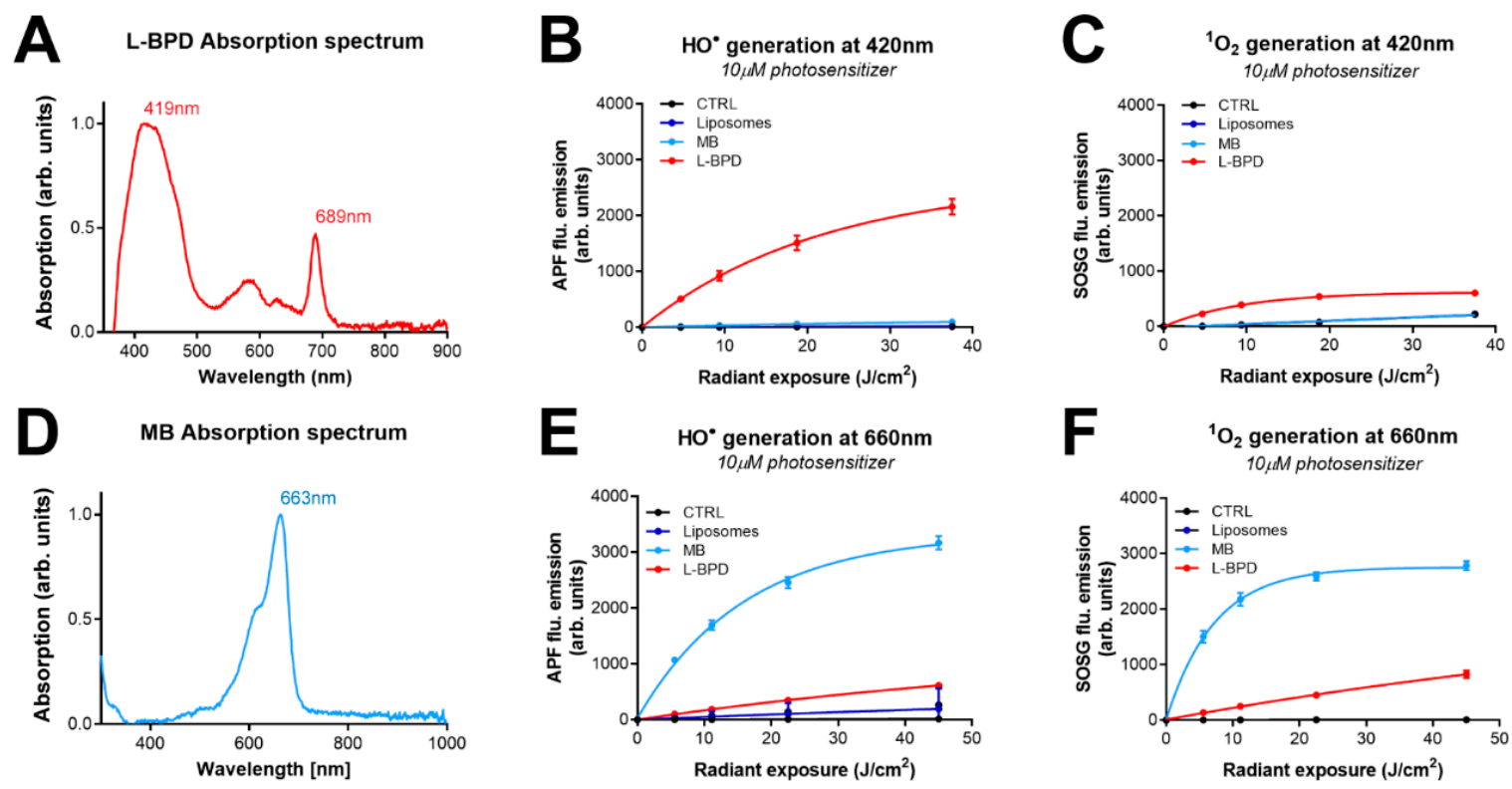

Figure 1. A characterization of the optical properties and photochemical activity of L-BPD and MB. (A) The absorption spectrum of L-BPD ( $40 \mu \mathrm{M}$ photosensitizer concentration in PBS), corrected for the absorption of empty liposomes at equimolar lipid concentrations. (B) Generation of $\mathrm{OH}^{\bullet}$ and (C) ${ }^{1} \mathrm{O}_{2}$ as upon exposure to $420 \mathrm{~nm}$ light, as measured by APF and SOSG fluorescence emission, respectively. Data represents the mean $\pm \mathrm{SD}$ of $\mathrm{N}=4$. (D) The absorption spectrum of $\mathrm{MB}(40 \mu \mathrm{M}$ photosensitizer concentration in PBS), corrected for the absorption of empty liposomes at equimolar lipid concentrations. (E) Generation of $\mathrm{OH}^{\bullet}$ and $(\mathbf{F}){ }^{1} \mathrm{O}_{2}$ as upon exposure to $660 \mathrm{~nm}$ light, as measured by APF and SOSG fluorescence emission, respectively. Data represents the mean \pm SD of $\mathrm{N}=4$. In all panels, black lines indicate control conditions (PBS), dark blue lines indicate empty liposomes (1.25 mM lipid), light blue lines indicate MB (10 $\mu \mathrm{M}$ in PBS), and red lines indicate L-BPD (10 $\mu \mathrm{M}$ BPD/1.25 mM lipid).

\subsection{The Dark Toxicity and Uptake of L-BPD and MB in Pancreatic Cancer Spheroids}

To define a suitable photosensitizer dose and incubation time before illumination, the dark toxicity and uptake of both photosensitizers were investigated. Since our different treatments may disintegrate the spheroid integrity, the detached cells could occupy a larger area, which is what is analyzed by our CALYPSO quantitative analysis. This is why it is very important not only to quantify the size/area of a spheroid, but also its viability and the extent of necrosis. In the dark, after $72 \mathrm{~h}$ of incubation and up to a photosensitizer concentration of $10 \mu \mathrm{M}$, neither the viability, spheroid size, nor the extent of necrosis was influenced in the PDAC spheroids (Figure 2). At $20 \mu \mathrm{M}$, a minor trend towards a lower spheroid size (MB, PANC-1 (Figure 2B)) and a lower viability (MB \& L-BPD, MIA PaCa-2 (Figure 2D,F)) was observed. Albeit minimal, findings suggest that both photosensitizers have negligible dark toxicity, and are well tolerated up to concentrations of $20 \mu \mathrm{M}$. 

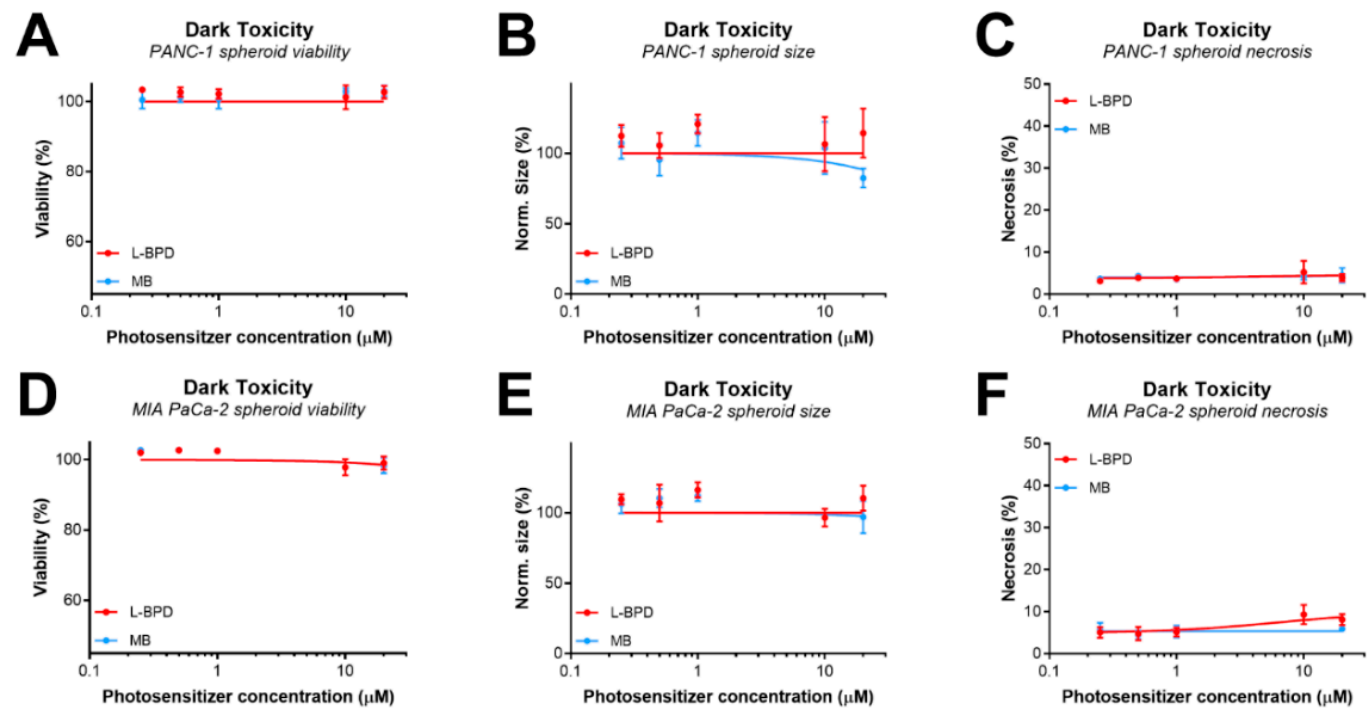

Figure 2. The dose-dependent dark toxicity of L-BPD and MB in PANC-1 (A-C) and MIA PaCa-2 spheroids (D-F). Toxicity was investigated based on the spheroid viability (A,D), spheroid size (B,E), and the extent of spheroid necrosis $(\mathbf{C}, \mathbf{F})$. The data depicts the mean $\pm \mathrm{SD}$ of the integrated data from four technical repeats $(\mathrm{N}=4-12)$.

Interestingly, the photosensitizer uptake in spheroids was dependent on both photosensitizer type and cell line (Figure 3). Using one phase association curve fit, we determined that the uptake half-time of L-BPD was 4.5 and $1.5 \mathrm{~h}$ for PANC-1 and MIA PaCa-2, respectively (Figure 3C). For MB, uptake half-time was 1.35 and $0.68 \mathrm{~h}$ for PANC-1 and MIA PaCa-2 spheroids (Figure 3D). Furthermore, by assessing the photosensitizer spatial distribution at a focal plane of $40 \mu \mathrm{m}$, we observed that L-BPD was homogeneously distributed within both PANC-1 and MIA PaCa-2 spheroids (Figure 3E,G). In contrast, the distribution of $\mathrm{MB}$ was highly heterogeneous, and localized in distinct foci within the spheroids (Figure 3F,H). The difference in uptake kinetics between PANC-1 and MIA PaCa-2 are likely explained by the different spheroid morphologies: rapid penetration occurs in the rather loose aggregates of MIA PaCa-2 cells, whereas the photosensitizers require more time to penetrate fully into the denser PANC-1 spheroids. In addition, the intrinsic properties of the different photosensitizer formulation may also play a role [26,38]. Based on the results, an incubation time of $4 \mathrm{~h}$ was selected for all further experiments.

\subsection{The Effect of Photosensitzer Dose on PDT Efficacy of L-BPD and MB in Pancreatic Cancer Spheroids}

Following the PDT settings described in Figure S1, we next evaluated the PDT efficacy of both photosensitizers. First, the PDT efficacy was investigated at $50 \mathrm{~J} / \mathrm{cm}^{2}$ with varying photosensitizer concentrations ranging from 0.2-20 $\mu \mathrm{M}$ (Figure 4). For any photosensitizer concentration, L-BPD was shown to be more efficient than MB. Inspection of the PANC-1 spheroid viability (Figure 4C) shows that both photosensitizers follow the same constant trend with a slight viability increase at high concentrations. Upon analysis of the necrosis data, no influence on necrosis was detected when changing the photosensitizer concentration (Figure 4E). Regarding the size of PANC-1 spheroids after PDT, no variation was observed with L-BPD. In contrast, a noticeable dose-response decrease was observed with MB (Figure 4D). Concerning MIA PaCa-2 spheroids, L-BPD did not exhibit photosensitizer dose-responses in terms of viability and necrosis, with the exception of the highest tested dose (Figure 4F,H). Finally, no dose-response could be determined regarding the size in both L-BPD and MB treated spheroids (Figure 4G). As a whole, the efficiency of L-BPD is higher at low concentrations, whereas MB efficiency increases at high concentrations. The data suggests that L-BPD treatment relies on necrosis for both PDAC spheroid models, 
whereas MB increases MIA PaCa-2 necrosis extent while its effect potentially relies on apoptosis and growth inhibition in PANC-1 spheroids.

A

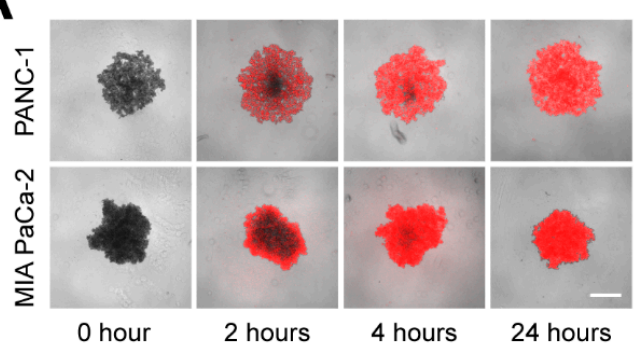

C

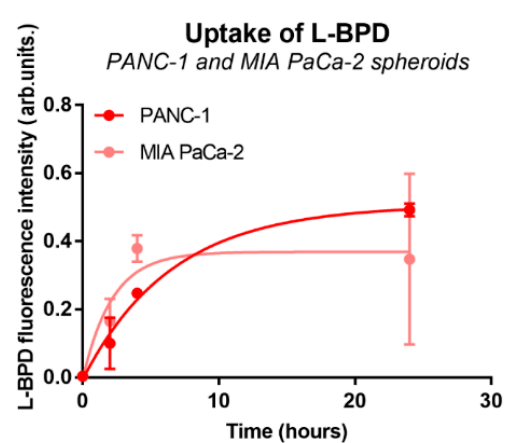

E

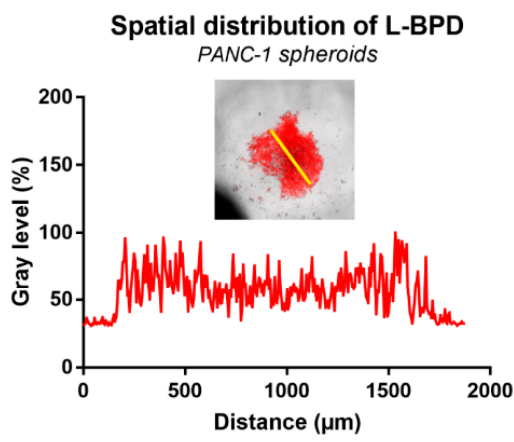

$\mathbf{G}$

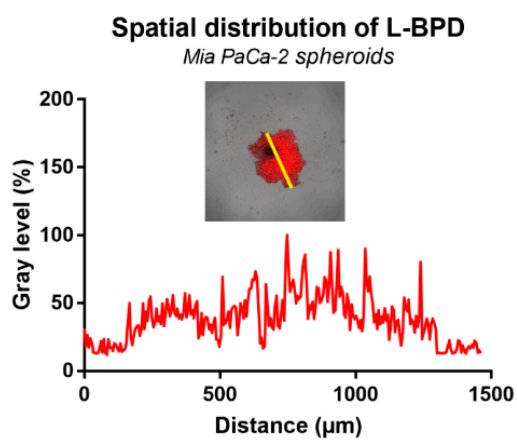

B

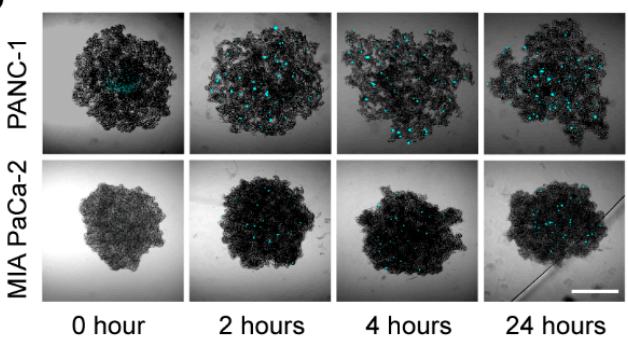

$\mathbf{D}$

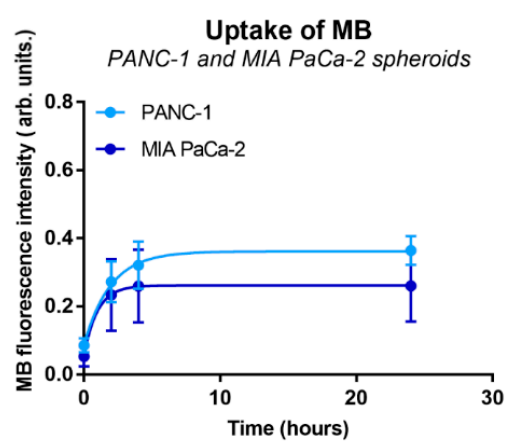

$\mathbf{F}$

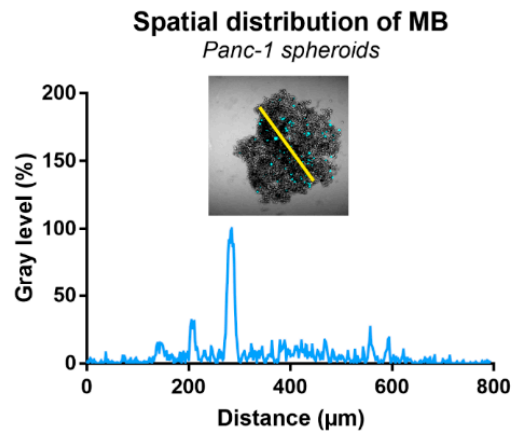

H

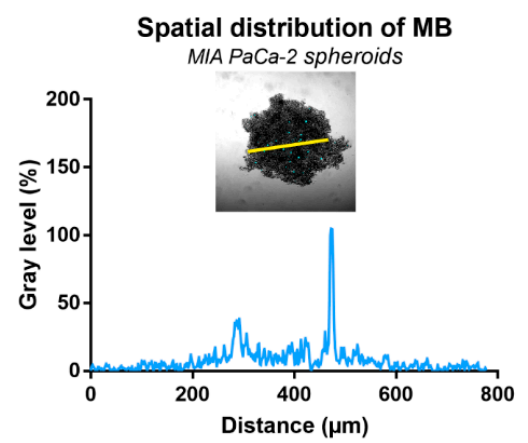

Figure 3. The time-dependent uptake of L-BPD $(\mathbf{A}, \mathbf{C}-\mathbf{E}, \mathbf{G})$ and $\mathrm{MB}(\mathbf{B}, \mathbf{D}-\mathbf{F}, \mathbf{H})$ in PANC-1 and MIA PaCa-2 spheroids. The fluorescence intensity of BPD ((A), red) and MB ((B), cyan) after 0, 2, 4 and $24 \mathrm{~h}$ of incubation was analyzed by quantitative microscopy $(\mathbf{G}, \mathbf{H})$. Data represents the mean $\pm \mathrm{SD}$ of $\mathrm{N}=6$ obtained from two technical repeats. To evaluate the spatial distribution of photosensitizer in the spheroids, L-BPD (C-G) and MB (D-H) fluorescence was quantified through longitudinal cross sections (yellow lines) of PANC-1 and MIA PaCa-2 spheroids. Scale bar $=500 \mu \mathrm{m}$. 
A

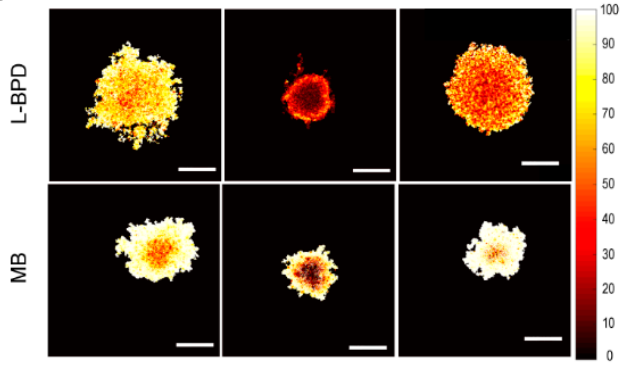

Light alone

PS $0.2 \mu \mathrm{M}$

PS $20 \mu \mathrm{M}$

B

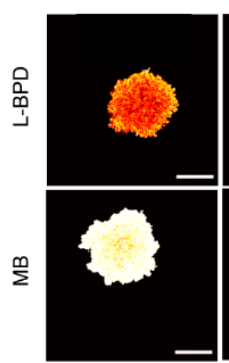

Light alone

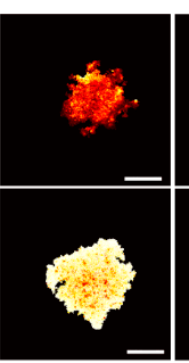

PS $0.2 \mu \mathrm{M}$

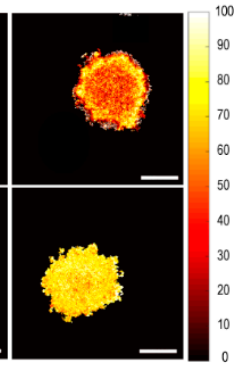

PS $20 \mu \mathrm{M}$
C

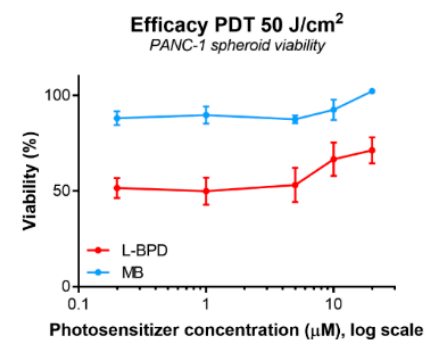

D

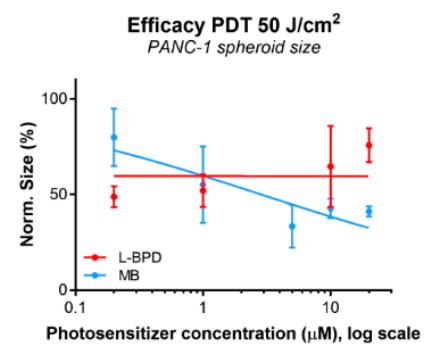

E

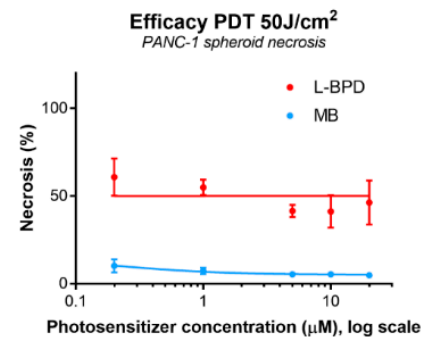

F

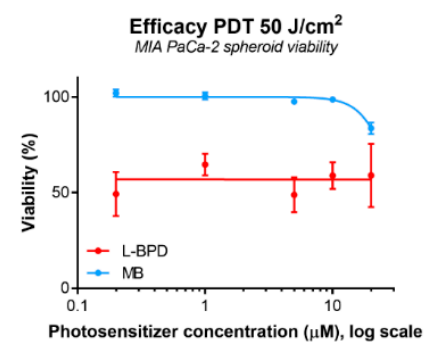

G

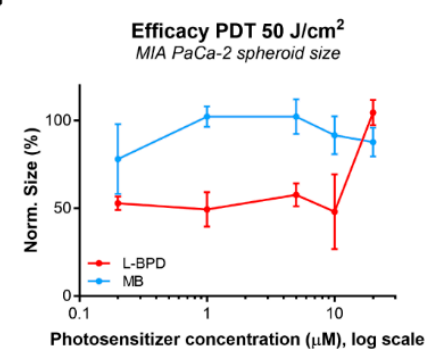

H

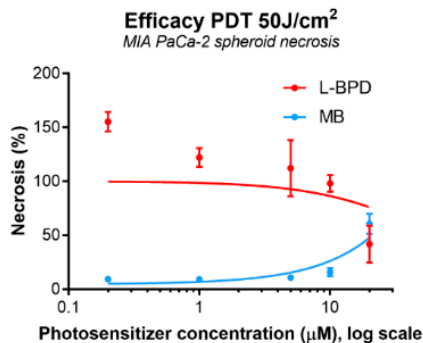

Figure 4. The effect of photosensitizer doses on the PDT efficacy $\left(50 \mathrm{~J} / \mathrm{cm}^{2}\right)$ of L-BPD and MB on PDAC spheroids. (A,B) Heatmaps of PANC-1 (A) and MIA PaCa-2 spheroids (B) following PDT with either L-BPD or MB at different photosensitizer doses. Scale bar $=500 \mu \mathrm{m}$. (C-H) The PDT efficacy was determined based on the spheroid viability $(\mathbf{C}, \mathbf{F})$, spheroid size $(\mathbf{D}, \mathbf{G})$, and the extent of spheroid necrosis $(\mathbf{E}, \mathbf{H})$, in relation to the photosensitizer dose. Data were depicted at the mean $\pm \mathrm{SD}$ of $\mathrm{N}=12$ obtained from three technical repeats. Dose responses curves were fitted using non-linear regression (inhibitor versus normalized response and antagonist versus normalized response).

We next evaluated the PDT-efficacy of L-BPD and MB at different radiant exposures. PDAC spheroids were incubated with either $0.2 \mu \mathrm{M}$ L-BPD or $20 \mu \mathrm{M} \mathrm{MB}$, exposed to radiant exposures in the range of $5-50 \mathrm{~J} / \mathrm{cm}^{2}$, after which the treatment efficacy was determined $72 \mathrm{~h}$ post-PDT (Figure 5). The photosensitizer concentrations were chosen based on the findings in Figure 4, which correlate well with the doses of previous studies [27,29]. PDT treatment has a similar impact with L-BPD in both cell lines, inducing significant reductions in spheroid viability at a dose of $50 \mathrm{~J} / \mathrm{cm}^{2}$ (Figure 5A,D). In contrast, the impact of MBPDT on spheroid viability is minimal; only a minor reduction is observed in MIA PaCa-2 spheroids (Figure 5D). Upon analyzing the spheroid size after PDT, the data suggests a comparable dose-dependent decrease in PANC-1 spheroids treated with L-BPD and MB (Figure 5B). In contrast, whereas the spheroid size also decreases in a light dose-dependent manner for L-BPD-PDT in MIA PaCa-2 spheroids, no influence on spheroid size was detected for MB-PDT (Figure 5E). Analysis of the extent of necrosis revealed a similar dose-response curve in PANC-1 spheroids as for the viability (Figure 5C). In MIA PaCa-2 
spheroids, the necrosis revealed a substantially stronger light dose response compared to the viability and size analysis (Figure 5F). Taken together, these findings demonstrate that the efficacy of PDT is not only dependent on the light dose (radiant exposure), but also on the photosensitizer. Moreover, the PDT efficacy manifests in different ways in distinct spheroids. For the PANC-1 spheroids, PDT with L-BPD reduces both cell viability and spheroid size, whereas the treatment MB-PDT reduces spheroid size but neither reduces viability nor increases necrosis. This difference between the effects of L-BPD and MB regarding size and viability may relate to different photosensitizer localization; extracellular L-BPD or intracellular localization to lysosomes and plasma membranes [27] may primarily induce necrosis in PANC-1 spheroids, whereas intracellular MB may be more efficient at inducing apoptosis or inhibiting spheroid growth. This is consistent with previous observations, demonstrating the nuclear localization of MB in cancer cell lines [39]. In MIA PaCa-2 spheroids, the organoid viability and normalized spheroid size demonstrated a similar dose-response correlation for L-BPD-PDT compared to PANC-1 spheroids. In contrast, the effects of MB were most clearly observed upon analysis of spheroid necrosis. A difference in sensitivity to nuclear oxidative damage between PANC-1 and MIA PaCa-2 cells may explain this discrepancy. Nonetheless, with both photosensitizers, a substantial treatment effect was observed at high total radiant exposures of $50 \mathrm{~J} / \mathrm{cm}^{2}$, which we thus selected as the optimal parameter in subsequent experiments.
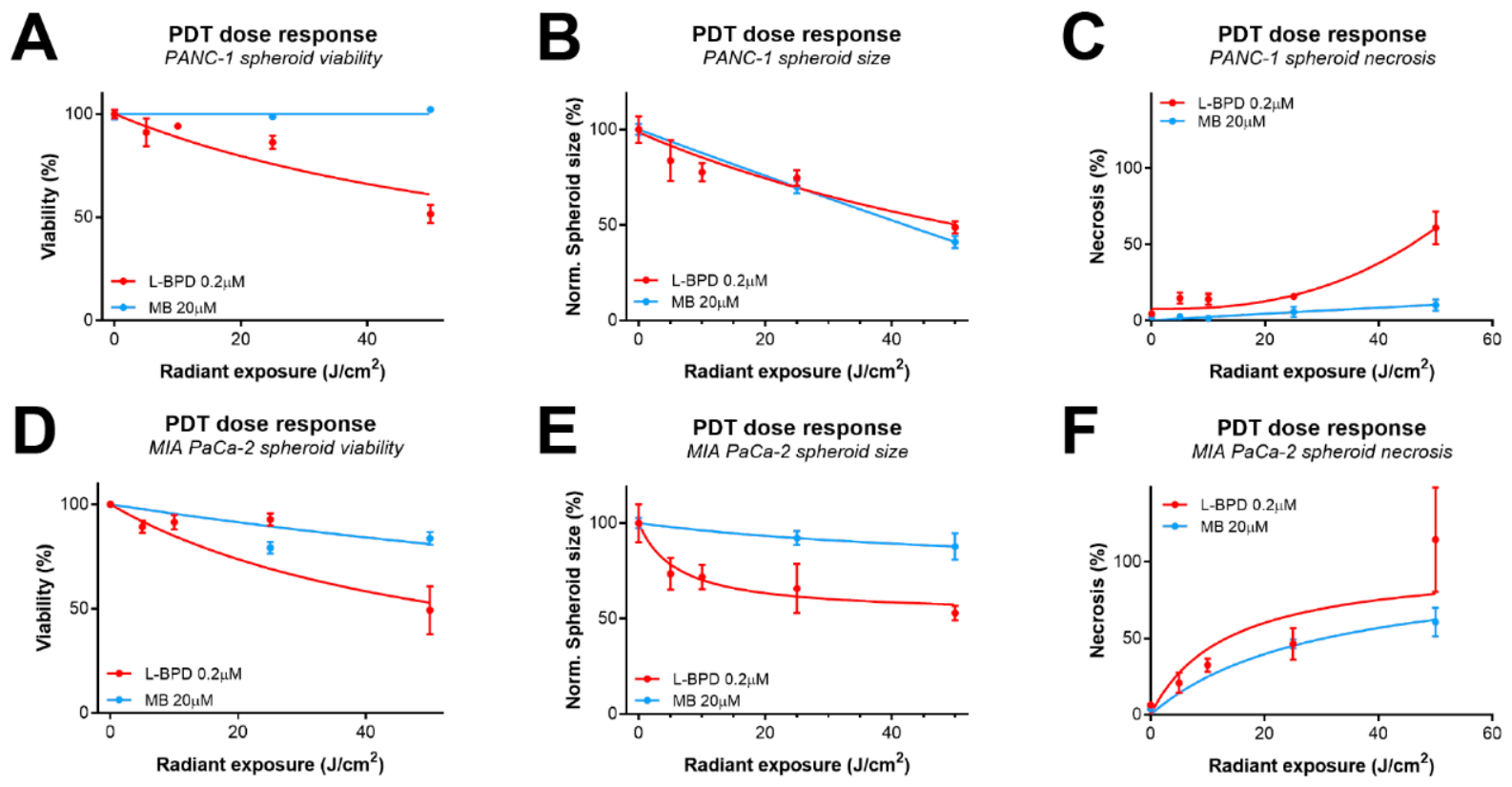

Figure 5. The effect of total radiant exposure (light fluence) on the PDT efficacy on PDAC spheroids. The PDT efficacy of L-BPD $(0.2 \mu \mathrm{M})$ and MB $(20 \mu \mathrm{M})$ was determined at different radiant exposures, and plotted based on the spheroid viability $(\mathbf{A}, \mathbf{D})$, spheroid size $(\mathbf{B}, \mathbf{E})$, and the extent of spheroid necrosis $(\mathbf{C}, \mathbf{F})$ in relation to the total radiant exposure. Data were depicted the mean $\pm \mathrm{SD}$ of $\mathrm{N}=9$, obtained from three technical repeats. Dose responses curves were fitted using non-linear regression (Inhibitor versus normalized response for viability and size data, and antagonist versus normalized response for necrosis data).

\subsection{The Effect of Fluence Rate on the PDT Efficacy of L-BPD and MB}

In the previous experiments, we observed a light dose-dependent PDT effect of L-BPD and $\mathrm{MB}$ on both PDAC spheroid models (Figure 5). Therefore, we next evaluated the efficacy of PDT with a total radiant exposure of $50 \mathrm{~J} / \mathrm{cm}^{2}$, given at different fluence rates: either low $\left(78 \mathrm{~mW} / \mathrm{cm}^{2}\right.$ for $420 \mathrm{~nm}, 93 \mathrm{~mW} / \mathrm{cm}^{2}$ for $\left.660 \mathrm{~nm}\right)$, medium $\left(171 \mathrm{~mW} / \mathrm{cm}^{2}\right.$ for $420 \mathrm{~nm}, 187 \mathrm{~mW} / \mathrm{cm}^{2}$ for $\left.660 \mathrm{~nm}\right)$, and high $\left(235 \mathrm{~mW} / \mathrm{cm}^{2}\right.$ for $420 \mathrm{~nm}, 280 \mathrm{~mW} / \mathrm{cm}^{2}$ for $660 \mathrm{~nm})$. 
First of all, heatmaps clearly indicate a toxicity of blue light $(420 \mathrm{~nm})$ when medium and high fluence rates are used, on both PANC-1 and MIA PaCa-2 spheroids (Figure 6A,B). However, no toxicity is observed for blue light at the low fluence rate, as well as for red light $(660 \mathrm{~nm})$ at low, medium, and high fluence rates.

A

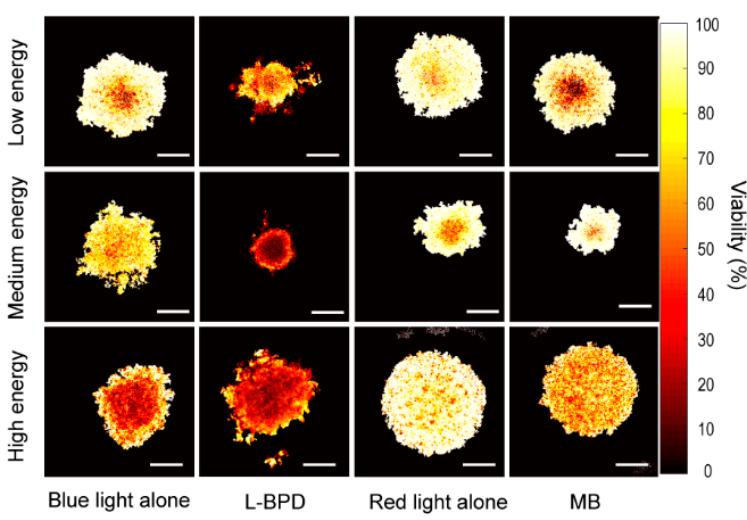

B

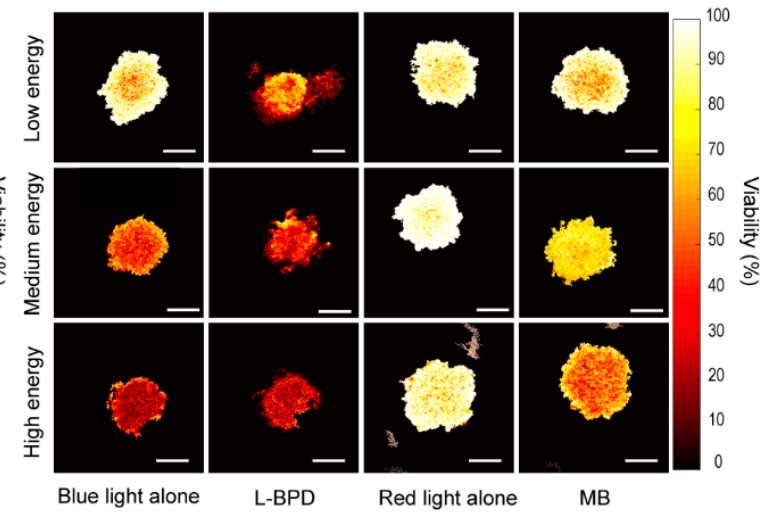

$E$
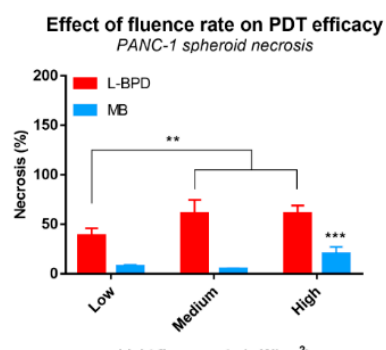

Light fluence rate $\left(\mathrm{mW} / \mathrm{cm}^{2}\right)$

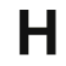

Effect of fluence rate on PDT efficacy Pace 2 spheroid necrosis

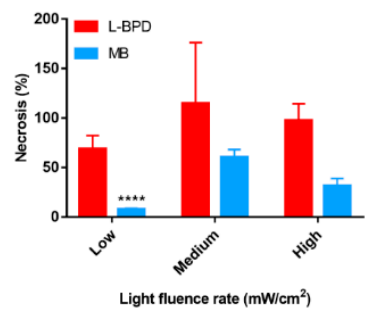

Figure 6. The effect of light energy on the PDT efficacy, given a total radiant exposure of $50 \mathrm{~J} / \mathrm{cm}^{2}$, on PDAC spheroids. (A,B) Heatmaps of PANC-1 (A) and MIA PaCa-2 spheroids (B) following PDT with either L-BPD or MB at different light energies. Scale bar $=500 \mu \mathrm{m}$. PDT effect using low $\left(78 \mathrm{~mW} / \mathrm{cm}^{2}\right.$ for L-BPD and $95 \mathrm{~mW} / \mathrm{cm}^{2}$ for MB), medium (170 $\mathrm{mW} / \mathrm{cm}^{2}$ for L-BPD and $190 \mathrm{~mW} / \mathrm{cm}^{2}$ for MB) or high $\left(235 \mathrm{~mW} / \mathrm{cm}^{2}\right.$ for L-BPD and $280 \mathrm{~mW} / \mathrm{cm}^{2}$ for MB) energies was investigated based on the spheroid viability $(\mathbf{C}, \mathbf{F})$, spheroid size $(\mathbf{D}, \mathbf{G})$, and the extent of spheroid necrosis $(\mathbf{E}, \mathbf{H})$. Data were depicted at the mean $\pm \mathrm{SD}$ of $\mathrm{N}=6$, obtained from two technical repeats. For each photosensitizer, results obtained with low, medium, and high energies were compared statistically by a Tukey's post hoc multiple comparisons test $\left(^{*}: p \leq 0.05 ;^{* *}: p \leq 0.01{ }^{* * *}: p \leq 0.0005 ;{ }^{* * * *}: p \leq 0.0001\right)$. Unless otherwise indicated, statistical differences with the other groups for each photosensitizer are indicated in the graphs.

In PANC-1 spheroids, a viability of $51.6 \pm 7.3 \%$ was observed using L-BPD irradiated at a fluence rate of $170 \mathrm{~mW} / \mathrm{cm}^{2}$, which is significantly lower than spheroids treated at low $\left(78 \mathrm{~mW} / \mathrm{cm}^{2}\right)$ and high $\left(235 \mathrm{~mW} / \mathrm{cm}^{2}\right)$ fluence rates (viabilities of $72.3 \pm 9.4 \%$ and $72.6 \pm 9.7 \%$ ) respectively. In MB-treated spheroids, we observed a significantly lower viability $(89.5 \pm 9.3 \%)$ when irradiation was performed at the highest fluence rate, in comparison to the low $(99.7 \pm 3.6 \%)$ and medium $(120.2 \pm 0.5 \%)$ fluence rates (Figure $6 \mathrm{C}$ ). 
These results are confirmed by quantifying the extent of necrosis, which was significantly higher when PANC-1 spheroids were treated with medium and high fluence rates for both photosensitizers (Figure 6E). In both L-BPD and MB treated PANC-1 spheroids, a statistically significant size reduction was observed following the irradiation at the intermediate fluence rate compared to low and high fluence rates (Figure 6D).

In MIA PaCa-2 spheroids, the viability following MB-PDT is significantly decreasing in a fluence rate-dependent manner, whereas conversely, viabilities of $41.8 \pm 13.9,47.9 \pm 17.6$, and $81.3 \pm 14.5 \%$ were observed with L-BPD-treated MIA PaCa-2 spheroids at low, medium, and high fluence rates respectively (Figure 6F). In terms of necrosis, for L-BPD-treated MIA PaCa-2 spheroids, no statistical differences were observed between the different fluence rates (Figure 6H). However, significantly higher necrosis was observed in MBtreated MIA PaCa2- spheroids illuminated at medium (60.6 $\pm 7.5 \%)$ and high $(31.4 \pm 7.5 \%)$ fluence rates, in comparison with spheroids treated at a low $(7.8 \pm 1.0 \%)$ fluence rate (Figure $6 \mathrm{H}$ ) confirming the results obtained in terms of viability. In L-BPD treated MIA $\mathrm{PaCa}-2$ spheroids, a significant size reduction is observed when a medium energy is applied, in comparison to spheroids treated with low and high fluence rates (Figure 6G). Finally, a significant reduction of MB-treated MIA PaCa-2 spheroid size was observed when the lower fluence rate was applied (Figure 6G).

Altogether, the results suggest that, in the case of L-BPD, the fluence rate is of minor impact in comparison to the total radiant exposure delivered to the photosensitizer. Indeed, whereas light dose-dependent PDT effects could be observed in terms of spheroid viability, size, and necrosis (Figure 4), no clear trends could be observed herein even if a mild fluence rate-dependent effect in terms of necrosis could be observed on PANC-1 spheroids (Figure 6E). However, $420 \mathrm{~nm}$ blue light, required for L-BPD excitation, clearly becomes cytotoxic at fluence rates $\geq 170 \mathrm{~mW} / \mathrm{cm}^{2}$ (Figure $6 \mathrm{~A}, \mathrm{~B}$ ), which is likely a photothermal effect. This effect complicates a clear assessment of the L-BPD PDT efficacy alone.

Concerning MB treatment, interesting fluence rate-dependent effects could be observed on PANC-1 spheroids in terms of viability, and on MIA PaCa-2 spheroids for both viability and extent of necrosis. Those results show that high photosensitizer concentrations, associated with high radiant exposures as well as high fluence rates, could be interesting for MB-PDT of spheroids. Nonetheless, L-BPD is generally more efficient than MB, even at doses that are 100 times lower (Figure 6), which confirms the results observed in the previous experiments.

\section{Discussion}

Although PDT is well-established as a potent cancer therapy, it remains a treatment with a large number of variables, ranging from pharmacological, physical, and biological parameters. The pharmacological parameters include the photosensitizer type, concentration, and uptake efficiency. As mentioned, nanoparticular formulations are increasingly used for improving the biocompatibility and cancer specificity of photosensitizers, and these may influence the pharmacological behavior of the included photosensitizers, including uptake efficiency and intratumor- and intracellular localization. Inspired by previous works in which various PDT dose parameters were systematically optimized for specific photosensitizers [18-20,23,24,28], we set out to determine the effects of the photophysical parameters of light fluence (radiant exposure) and fluence rate of L-BPD and MB, which represent two widely used photosensitizers. We evaluated the application of two stateof-the-art 96-well LED arrays for the treatment of cancer spheroids with PDT. A $420 \mathrm{~nm}$ violet light array was selected as it overlaps with the Soret band of a plethora of porphyrins, and may thus be useful as a versatile excitation source for this class of photosensitizers. A second array of $660 \mathrm{~nm}$ was selected as it overlaps with the absorption peak of various photosensitizers, such as phthalocyanines, chlorins, and MB.

Regarding the efficacy of L-BPD, we demonstrate that L-BPD is a potent photosensitizer upon $420 \mathrm{~nm}$ excitation, with limited dark toxicity and good spheroid penetration. In further studies on L-BPD, our findings suggest using a drug-light interval of at least $4 \mathrm{~h}$, 
and BPD concentrations in the range of $0.1-1 \mu \mathrm{M}$. The PDT efficacy is then best investigated as a function of total radiant exposure. Nonetheless, great care must be taken to avoid any phototoxic effects of the $420 \mathrm{~nm}$ excitation light alone by not exceeding fluence rates of $73 \mathrm{~mW} / \mathrm{cm}^{2}$. These results correlate well to previous findings. A study by Rizvi et al. demonstrated that PDT with a dose of $0.25 \mu \mathrm{M}$ was more effective than 1 and $10 \mu \mathrm{M}$ in 3D culture models of ovarian cancer. It was also shown that at a dose of $10 \mu \mathrm{M}$, the fluence rate was inversely related to treatment efficacy. Moreover, when trying to produce the same cytotoxic response using $10 \mu \mathrm{M}$ of BPD, a reduction in fluence rate from 150 to $0.5 \mathrm{~mW} / \mathrm{cm}^{2}$ was necessary [28].

Regarding $\mathrm{MB}$, our findings show that $\mathrm{MB}$ is a rather poor photosensitizer for PDT of cancer spheroids, despite showing excellent PDT efficacy in cell-free assays. This is likely a result of poor spheroid uptake and aggregation. Treatment with doses exceeding $20 \mu \mathrm{M}$ is possible due to the low dark toxicity of $\mathrm{MB}$, yet it is not clear if this will lead to a better uptake and more homogeneous intratumor distribution. Our findings correlate relatively well to various previous studies. The efficacy of MB has previously been evaluated in lung cancer cell lines, in which a MB dose of $160 \mu \mathrm{M}$, and a radiant exposure of $95 \mathrm{~J} / \mathrm{cm}^{2}$ $(660 \mathrm{~nm})$ was applied. Although light alone was non-toxic, MB alone had significant dark toxicity, inducing $>50 \%$ cell death. The combination, i.e., MB-PDT, was effective with a $95 \%$ reduction in viability. It should be mentioned that the fluence rate could not be retrieved. Despite poor in vitro findings, in vivo findings have demonstrated that MB holds potential for PDT of solid cancers. In a subcutaneous mouse xenograft model of colorectal cancer, $\mathrm{MB}$ was shown effective at a dose of $100 \mathrm{~J} / \mathrm{cm}^{2}(660 \mathrm{~nm})$. Decreasing the fluence rate from 100 to $50 \mathrm{~mW} / \mathrm{cm}^{2}$ resulted in a significantly increased PDT efficacy [34]. However, it is worth noting that those results were obtained upon intratumor injection, which remove the issues linked to the uptake of MB. Similar results were obtained in subcutaneous lung carcinoma xenografts in mice and in a hamster model of oral cancer [33]. PDT was shown effective at a radiant exposure of $200 \mathrm{~J} / \mathrm{cm}^{2}$. In both cases, $50-150 \mu \mathrm{L}$ of a $1 \% w / v(31.3 \mathrm{mM})$ solution of MB was injected, confirming the need for high MB doses for effective PDT. The poor performance of $\mathrm{MB}$ may suggest that nanoparticular encapsulation may be explored to improve its uptake and efficacy for the treatment of cancer [32].

Aspects that were not investigated for L-BPD and MB in this study include the druglight interval and continuous versus fractionated PDT regiments. We selected the drug-light interval based on the uptake assays described in Figure 3, demonstrating near-maximal photosensitizer accumulation after $4 \mathrm{~h}$ of incubation. For subsequent experiments, we assumed that the higher the tumor accumulation of the photosensitizer, the higher the PDT efficacy. However, it has been shown that the intracellular localization of photosensitizers changes over time, and this may affect the PDT efficacy [26]. Moreover, we and others [28] have shown that higher photosensitizer doses, especially for (L-)BPD, do not necessarily improve treatment outcomes. At high concentrations, BPD may be quenched and exhibit lower PDT efficacy, which may explain the lack of a clear photosensitizer-dose response in Figure 4. Regarding continuous versus fractionated PDT regimens, a multitude of comparisons are possible, which is why such parameters were not included in this study. Benefits of a fractionated regime (e.g., twice $25 \mathrm{~J} / \mathrm{cm}^{2}$ ) versus a continuous irradiation (once $50 \mathrm{~J} / \mathrm{cm}^{2}$ ) includes the re-oxygenation of the cancer tissues during the fractionated regime $[19,23]$. Another benefit may be the reduction of photothermal effects during prolonged irradiation, which may be especially relevant for irradiation with blue light. The phototoxic effects of blue light in absence of PDT are an additional and strong rationale for using $690 \mathrm{~nm}$ light for the excitation of (L-)BPD. Ongoing studies will compare the impact of these factors (drug-light interval, fractionation, blue versus red light) on the PDT efficacy.

Although three dimensional culture models are promising models that bridge the gap between in vitro and in vivo experimentation in translational oncology [40]; our study utilizes spheroids as a rather simple 3D culture model. The effects of the cancer stroma, which are especially important for pancreatic cancer, are not taken into account. Previous studies have shown that addition of fibroblasts to PDAC spheroids induces spheroid compression 
and may greatly limit the diffusion of therapeutics into the spheroid masses [21,41-43]. Similar observations have been made on heterotypic microtumors in which cancer cells were co-cultured with cancer-associated fibroblasts. Thus, although the effects of the photophysical parameters defined in this study may be universally applied to spheroid and organoid models, the pharmacological variables (photosensitizer dose and drug-light interval) likely require optimization in a model-dependent manner. Finally, it is worth noting that within 3D culture models there is substantial heterogeneity between cells in terms of drug exposure, as well as nutrient and oxygen gradients that can influence treatment susceptibility [40,44-47]. In this study, we have assessed the effects of PDT on a more macroscopic level using in situ staining and low-magnification microscopy, which does not take into account any effects on the individual cell level. Whereas the dissociation of spheroids and organoids to detect modes of cell death by flow cytometry is technically feasible, the procedure itself it can lead to substantial amounts of cell death [41], which challenges the accuracy of single cell analyses such as flow cytometry. Flow cytometry is thus best used for 2D cell cultures, where obtaining single-cell suspensions is less problematic. Nonetheless, annexin $\mathrm{V}$ and propidium iodide staining in situ may be feasible, as was recently shown by Karimnia et al. [48]. High resolution confocal microscopy, combined with optical clearing, may be a more promising method to study treatment effects on the individual cell levels within 3D cancer models [49].

\section{Conclusions}

The efficacy of photosensitizers for the treatment of cancer with PDT requires a careful optimization of the PDT dose parameters. This study clearly demonstrates that variations in the photosensitizer type, photosensitizer dose, light dose, and the light dose-rate have a strong impact on the overall PDT efficacy in 3D culture models of pancreatic cancer. The optimization of these parameters for fundamental and translational studies should be carefully performed, and the publication of such findings should be encouraged to expedite research and development towards new light sources, photosensitizers, and nanoformulations of photosensitizers for PDT.

Supplementary Materials: The following supporting information can be downloaded at: https: / / www.mdpi.com/article/10.3390/onco2010002/s1, Figure S1: Photodynamic therapy settings for the treatment of pancreatic cancer spheroids by either MB or L-BPD.

Author Contributions: Conceptualization, T.L.C. and M.B.; Data Curation, N.M.C.G., N.P.-S. and M.B.; Formal Analysis, T.L.C., N.M.C.G. and M.B.; Funding Acquisition, M.B.; Investigation, T.L.C., N.M.C.G., N.P.-S., J.-L.C. and M.B.; Methodology, T.L.C., N.M.C.G., N.P.-S. and M.B.; Project Administration, M.B.; Resources, J.-L.C. and M.B.; Supervision, T.L.C., J.-L.C. and M.B.; Validation, T.L.C. and M.B.; Visualization, T.L.C., N.M.C.G. and M.B.; Writing-Original Draft, T.L.C., N.M.C.G., N.P.-S., J.-L.C. and M.B.; Writing-Review and Editing, T.L.C., N.M.C.G., N.P.-S., J.-L.C. and M.B. All authors have read and agreed to the published version of the manuscript.

Funding: This research was funded by the Phospholipid Research Center, Heidelberg, Germany, grant number MAB-2020-080/1-1 and the Institut National de la Santé et de la Recherche Medicale (INSERM, Paris France).

Institutional Review Board Statement: Not applicable.

Informed Consent Statement: Not applicable.

Data Availability Statement: All data is available upon request.

Acknowledgments: The authors are grateful to Anne-Laure Bulin (Synchrotron Radiation for Biomedicine, INSERM, Grenoble-Alpes University, Grenoble, France) for her help with the quantitative image analysis.

Conflicts of Interest: The authors declare no conflict of interest. 


\section{References}

1. Christensen, E.; Warloe, T.; Kroon, S.; Funk, J.; Helsing, P.; Soler, A.M.; Stang, H.J.; Vatne, O.; Mørk, C. Norwegian Photodynamic Therapy (PDT) Group Guidelines for Practical Use of MAL-PDT in Non-Melanoma Skin Cancer. J. Eur. Acad. Dermatol. Venereol. 2010, 24, 505-512. [CrossRef]

2. Hopper, C.; Niziol, C.; Sidhu, M. The Cost-Effectiveness of Foscan Mediated Photodynamic Therapy (Foscan-PDT) Compared with Extensive Palliative Surgery and Palliative Chemotherapy for Patients with Advanced Head and Neck Cancer in the UK. Oral Oncol. 2004, 40, 372-382. [CrossRef]

3. Meulemans, J.; Delaere, P.; Vander Poorten, V. Photodynamic Therapy in Head and Neck Cancer: Indications, Outcomes, and Future Prospects. Curr. Opin. Otolaryngol. Head Neck Surg. 2019, 27, 136-141. [CrossRef]

4. Ohtani, K.; Ikeda, N. Photodynamic Therapy for Lung Cancer. Kyobu Geka 2016, 69, 694-699.

5. Usuda, J.; Kato, H.; Okunaka, T.; Furukawa, K.; Tsutsui, H.; Yamada, K.; Suga, Y.; Honda, H.; Nagatsuka, Y.; Ohira, T.; et al. Photodynamic Therapy (PDT) for Lung Cancers. J. Thorac. Oncol. 2006, 1, 489-493. [CrossRef]

6. Gheewala, T.; Skwor, T.; Munirathinam, G. Photosensitizers in Prostate Cancer Therapy. Oncotarget 2017, 8, $30524-30538$. [CrossRef] [PubMed]

7. Windahl, T.; Andersson, S.O.; Lofgren, L. Photodynamic Therapy of Localised Prostatic Cancer. Lancet 1990, 336, 1139. [CrossRef]

8. Yano, T.; Wang, K.K. Photodynamic Therapy for Gastrointestinal Cancer. Photochem. Photobiol. 2020, 96, 517-523. [CrossRef] [PubMed]

9. Pereira, S. Photodynamic Therapy for Pancreatic and Biliary Tract Cancer: The United Kingdom Experience. J. Natl. Compr. Canc. Netw. 2012, 10 (Suppl. 2), S48-S51. [CrossRef]

10. Huggett, M.T.; Jermyn, M.; Gillams, A.; Illing, R.; Mosse, S.; Novelli, M.; Kent, E.; Bown, S.G.; Hasan, T.; Pogue, B.W.; et al. Phase I/II Study of Verteporfin Photodynamic Therapy in Locally Advanced Pancreatic Cancer. Br. J. Cancer 2014, 110, 1698-1704. [CrossRef]

11. Celli, J.P.; Solban, N.; Liang, A.; Pereira, S.P.; Hasan, T. Verteporfin-Based Photodynamic Therapy Overcomes Gemcitabine Insensitivity in a Panel of Pancreatic Cancer Cell Lines. Lasers Surg. Med. 2011, 43, 565-574. [CrossRef] [PubMed]

12. Fan, B.-G.; Andrén-Sandberg, A. Photodynamic Therapy for Pancreatic Cancer. Pancreas 2007, 34, 385-389. [CrossRef]

13. Banerjee, S.M.; El-Sheikh, S.; Malhotra, A.; Mosse, C.A.; Parker, S.; Williams, N.R.; MacRobert, A.J.; Hamoudi, R.; Bown, S.G.; Keshtgar, M.R. Photodynamic Therapy in Primary Breast Cancer. J. Clin. Med. 2020, 9, 483. [CrossRef]

14. Castano, A.P.; Demidova, T.N.; Hamblin, M.R. Mechanisms in Photodynamic Therapy: Part One-Photosensitizers, Photochemistry and Cellular Localization. Photodiagnosis Photodyn. Ther. 2004, 1, 279-293. [CrossRef]

15. Xu, S.; Bulin, A.-L.; Hurbin, A.; Elleaume, H.; Coll, J.-L.; Broekgaarden, M. Photodynamic Diagnosis and Therapy for Peritoneal Carcinomatosis: Emerging Perspectives. Cancers 2020, 12, 2491. [CrossRef]

16. Dolmans, D.E.J.G.J.; Fukumura, D.; Jain, R.K. Photodynamic Therapy for Cancer. Nat. Rev. Cancer 2003, 3, 380-387. [CrossRef]

17. Chen, Q.; Chopp, M.; Dereski, M.O.; Wilson, B.C.; Patterson, M.S.; Schreiber, A.; Hetzel, F.W. The Effect of Light Fluence Rate in Photodynamic Therapy of Normal Rat Brain. Radiat. Res. 1992, 132, 120-123. [CrossRef] [PubMed]

18. Henderson, B.W.; Busch, T.M.; Snyder, J.W. Fluence Rate as a Modulator of PDT Mechanisms. Lasers Surg. Med. 2006, 38, 489-493. [CrossRef]

19. Iinuma, S.; Schomacker, K.T.; Wagnieres, G.; Rajadhyaksha, M.; Bamberg, M.; Momma, T.; Hasan, T. In Vivo Fluence Rate and Fractionation Effects on Tumor Response and Photobleaching: Photodynamic Therapy with Two Photosensitizers in an Orthotopic Rat Tumor Model. Cancer Res. 1999, 59, 6164-6170.

20. Foster, T.H.; Hartley, D.F.; Nichols, M.G.; Hilf, R. Fluence Rate Effects in Photodynamic Therapy of Multicell Tumor Spheroids. Cancer Res. 1993, 53, 1249-1254.

21. Broekgaarden, M.; Anbil, S.; Bulin, A.-L.; Obaid, G.; Mai, Z.; Baglo, Y.; Rizvi, I.; Hasan, T. Modulation of Redox Metabolism Negates Cancer-Associated Fibroblasts-Induced Treatment Resistance in a Heterotypic 3D Culture Platform of Pancreatic Cancer. Biomaterials 2019, 222, 119421. [CrossRef]

22. Broekgaarden, M.; Weijer, R.; van Gulik, T.M.; Hamblin, M.R.; Heger, M. Tumor Cell Survival Pathways Activated by Photodynamic Therapy: A Molecular Basis for Pharmacological Inhibition Strategies. Cancer Metastasis Rev. 2015, 34, 643-690. [CrossRef]

23. Babilas, P.; Schacht, V.; Liebsch, G.; Wolfbeis, O.S.; Landthaler, M.; Szeimies, R.-M.; Abels, C. Effects of Light Fractionation and Different Fluence Rates on Photodynamic Therapy with 5-Aminolaevulinic Acid in Vivo. Br. J. Cancer 2003, 88, 1462-1469. [CrossRef]

24. Angell-Petersen, E.; Spetalen, S.; Madsen, S.J.; Sun, C.-H.; Peng, Q.; Carper, S.W.; Sioud, M.; Hirschberg, H. Influence of Light Fluence Rate on the Effects of Photodynamic Therapy in an Orthotopic Rat Glioma Model. J. Neurosurg. 2006, 104, $109-117$. [CrossRef]

25. Weijer, R.; Broekgaarden, M.; Kos, M.; van Vught, R.; Rauws, E.A.J.; Breukink, E.; van Gulik, T.M.; Storm, G.; Heger, M. Enhancing Photodynamic Therapy of Refractory Solid Cancers: Combining Second-Generation Photosensitizers with Multi-Targeted Liposomal Delivery. J. Photochem. Photobiol. C Photochem. Rev. 2015, 23, 103-131. [CrossRef]

26. Broekgaarden, M.; de Kroon, A.I.P.M.; Gulik, T.M.; van Heger, M. Development and in Vitro Proof-of-Concept of Interstitially Targeted Zinc- Phthalocyanine Liposomes for Photodynamic Therapy. Curr. Med. Chem. 2014, 21, 377-391. [CrossRef] [PubMed] 
27. Obaid, G.; Broekgaarden, M.; Bulin, A.-L.; Huang, H.-C.; Kuriakose, J.; Liu, J.; Hasan, T. Photonanomedicine: A Convergence of Photodynamic Therapy and Nanotechnology. Nanoscale 2016, 8, 12471-12503. [CrossRef] [PubMed]

28. Rizvi, I.; Anbil, S.; Alagic, N.; Celli, J.; Celli, J.P.; Zheng, L.Z.; Palanisami, A.; Glidden, M.D.; Pogue, B.W.; Hasan, T. PDT Dose Parameters Impact Tumoricidal Durability and Cell Death Pathways in a 3D Ovarian Cancer Model. Photochem. Photobiol. 2013, 89, 942-952. [CrossRef]

29. Jiang, C.; Yang, W.; Wang, C.; Qin, W.; Ming, J.; Zhang, M.; Qian, H.; Jiao, T. Methylene Blue-Mediated Photodynamic Therapy Induces Macrophage Apoptosis via ROS and Reduces Bone Resorption in Periodontitis. Oxid. Med. Cell. Longev. 2019, 2019, 1529520. [CrossRef]

30. Späth, A.; Leibl, C.; Cieplik, F.; Lehner, K.; Regensburger, J.; Hiller, K.-A.; Bäumler, W.; Schmalz, G.; Maisch, T. Improving Photodynamic Inactivation of Bacteria in Dentistry: Highly Effective and Fast Killing of Oral Key Pathogens with Novel Tooth-Colored Type-II Photosensitizers. J. Med. Chem. 2014, 57, 5157-5168. [CrossRef]

31. Shen, J.J.; Jemec, G.B.E.; Arendrup, M.C.; Saunte, D.M.L. Photodynamic Therapy Treatment of Superficial Fungal Infections: A Systematic Review. Photodiagnosis Photodyn. Ther. 2020, 31, 101774. [CrossRef]

32. Jesus, V.P.S.; Raniero, L.; Lemes, G.M.; Bhattacharjee, T.T.; Caetano Júnior, P.C.; Castilho, M.L. Nanoparticles of Methylene Blue Enhance Photodynamic Therapy. Photodiagnosis Photodyn. Ther. 2018, 23, 212-217. [CrossRef]

33. Dos Santos, A.F.; Terra, L.F.; Wailemann, R.A.M.; Oliveira, T.C.; Vinícius de Morais, G.; Mineiro, M.F.; Meotti, F.C.; Bruni-Cardoso, A.; Baptista, M.S.; Labriola, L. Methylene Blue Photodynamic Therapy Induces Selective and Massive Cell Death in Human Breast Cancer Cells. BMC Cancer 2017, 17, 194. [CrossRef]

34. Methylene Blue Mediated Photodynamic Therapy in Experimental Colorectal Tumors in Mice-PubMed. Available online: https:/ / pubmed.ncbi.nlm.nih.gov/11154085/ (accessed on 29 November 2021).

35. Carioli, G.; Malvezzi, M.; Bertuccio, P.; Boffetta, P.; Levi, F.; La Vecchia, C.; Negri, E. European Cancer Mortality Predictions for the Year 2021 with Focus on Pancreatic and Female Lung Cancer. Ann. Oncol. 2021, 32, 478-487. [CrossRef]

36. Bulin, A.-L.; Broekgaarden, M.; Hasan, T. Comprehensive High-Throughput Image Analysis for Therapeutic Efficacy of Architecturally Complex Heterotypic Organoids. Sci. Rep. 2017, 7, 16645. [CrossRef]

37. Price, M.; Reiners, J.J.; Santiago, A.M.; Kessel, D. Monitoring Singlet Oxygen and Hydroxyl Radical Formation with Fluorescent Probes during Photodynamic Therapy. Photochem. Photobiol. 2009, 85, 1177-1181. [CrossRef]

38. Bacellar, I.O.L.; Tsubone, T.M.; Pavani, C.; Baptista, M.S. Photodynamic Efficiency: From Molecular Photochemistry to Cell Death Int. J. Mol. Sci. 2015, 16, 20523-20559. [CrossRef] [PubMed]

39. Feng, X.; Jermain, P.; Muzikansky, A.; Ross, A.; Hamblin, M.; Yaroslavsky, A.N.; Yaroslavsky, A.N. Quantifying Subcellular Localization of Methylene Blue in Cultured Brain Cells. In Proceedings of the Frontiers in Optics + Laser Science APS/DLS (2019), Washington, DC, USA, 15-19 September 2019; Optical Society of America: Washington, DC, USA, 2019; p. JTu3A.97.

40. Pampaloni, F.; Reynaud, E.G.; Stelzer, E.H.K. The Third Dimension Bridges the Gap between Cell Culture and Live Tissue. Nat Rev. Mol. Cell. Biol. 2007, 8, 839-845. [CrossRef] [PubMed]

41. Bulin, A.-L.; Broekgaarden, M.; Simeone, D.; Hasan, T. Low Dose Photodynamic Therapy Harmonizes with Radiation Therapy to Induce Beneficial Effects on Pancreatic Heterocellular Spheroids. Oncotarget 2019, 10, 2625-2643. [CrossRef]

42. Broekgaarden, M.; Alkhateeb, A.; Bano, S.; Bulin, A.-L.; Obaid, G.; Rizvi, I.; Hasan, T. Cabozantinib Inhibits Photodynamic Therapy-Induced Auto- and Paracrine MET Signaling in Heterotypic Pancreatic Microtumors. Cancers 2020, 12, 1401. [CrossRef] [PubMed]

43. Priwitaningrum, D.L.; Blondé, J.-B.G.; Sridhar, A.; van Baarlen, J.; Hennink, W.E.; Storm, G.; Le Gac, S.; Prakash, J. Tumor StromaContaining 3D Spheroid Arrays: A Tool to Study Nanoparticle Penetration. J. Control. Release 2016, 244, 257-268. [CrossRef] [PubMed]

44. Glidden, M.D.; Celli, J.P.; Massodi, I.; Rizvi, I.; Pogue, B.W.; Hasan, T. Image-Based Quantification of Benzoporphyrin Derivative Uptake, Localization, and Photobleaching in 3D Tumor Models, for Optimization of PDT Parameters. Theranostics 2012, 2, 827-839. [CrossRef]

45. Broekgaarden, M.; Bulin, A.-L.; Porret, E.; Musnier, B.; Chovelon, B.; Ravelet, C.; Sancey, L.; Elleaume, H.; Hainaut, P.; Coll, J.-L.; et al. Surface functionalization of gold nanoclusters with arginine: A trade-off between microtumor uptake and radiotherapy enhancement. Nanoscale 2020, 12, 6959-6963. [CrossRef] [PubMed]

46. Etcheverry, M.E.; Pasquale, M.A.; Bergna, C.; Ponzinibbio, C.; Garavaglia, M. Photodynamic therapy in 2D and 3D human cervical carcinoma cell cultures employing LED light sources emitting at different wavelengths. Phys. Med. Biol. 2020, 65, 015017. [CrossRef] [PubMed]

47. Alderden, R.A.; Mellor, H.R.; Modok, S.; Hall, M.D.; Sutton, S.R.; Newville, M.G.; Callaghan, R.; Hambley, T.W. Elemental tomography of cancer-cell spheroids reveals incomplete uptake of both platinum(II) and platinum(IV) complexes. J. Am. Chem. Soc. 2007, 129, 13400-13401. [CrossRef]

48. Karimnia, V.; Rizvi, I.; Slack, F.J.; Celli, J.P. Photodestruction of Stromal Fibroblasts Enhances Tumor Response to PDT in 3D Pancreatic Cancer Coculture Models. Photochem. Photobiol. 2021, 97, 416-426. [CrossRef] [PubMed]

49. Dekkers, J.F.; Alieva, M.; Wellens, L.M.; Ariese, H.C.R.; Jamieson, P.R.; Vonk, A.M.; Amatngalim, G.D.; Hu, H.; Oost, K.C.; Snippert, H.J.G.; et al. High-resolution 3D imaging of fixed and cleared organoids. Nat. Protoc. 2019, 14, 1756-1771. [CrossRef] [PubMed] 